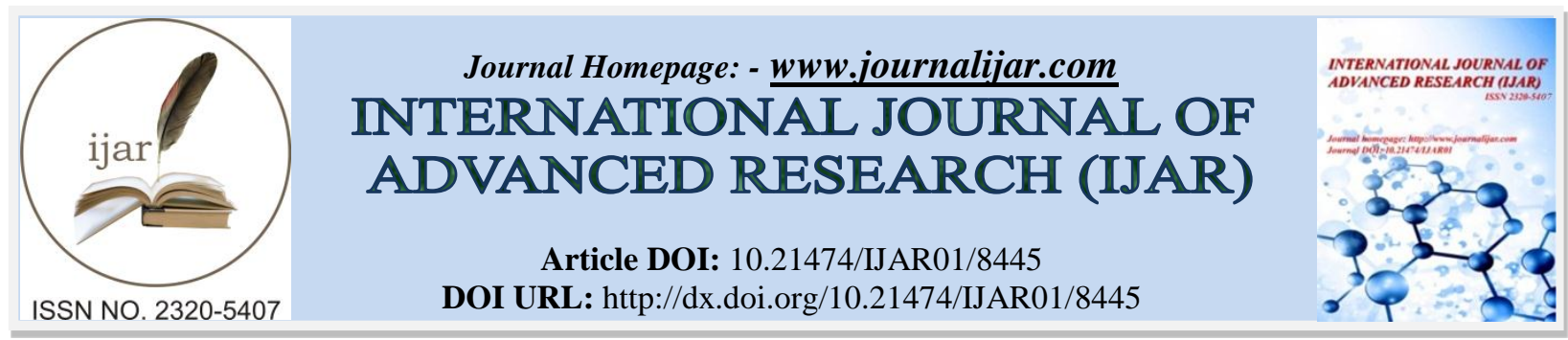

RESEARCH ARTICLE

\title{
COMPARISON BETWEEN THE CT \& MRI FOR MENINGIOMA IN THE BRAIN IN KING KHALID UNIVERSITY HOSPITAL RIYADH K.S.A.
}

Hisham Al-nutayfi ${ }^{1}$, Farah Masaad ${ }^{2}$, Abdullah Hamdan ${ }^{3}$, Nasraldeen Alnaeem Alkhidir ${ }^{3}$ and Sajna Ashraf ${ }^{4}$.

1. X-ray department, Al-Aflaj General Hospital M.O.H, Kingdom of Saudi Arabia.

2. Department of Oral \&Maxillofacial Radiology, Al-Farabi College of Dentistry and Nursing, Riyadh, Kingdom of Saudi Arabia.

3. College of Applied Medical Science, Department of Radiologic Technology Qassim University, Buraydah ., Kingdom of Saudi Arabia.

4. Department of oral \&Maxillofacial Radiology Al-Farabi College of Dentistry and Nursing, Riyadjh, Kingdom of Saudi Arabia.

\section{Manuscript Info}

Manuscript History

Received: 20 November 2018

Final Accepted: 22 December 2018

Published: January 2019

Key words:-

Meningioma and CT scan examination plus MRI. Chi-Square.

\section{Abstract}

Purpose: To determine the diagnostic accuracy values of CT. Scan in the detection of meningioma in the brain. Correlated the accuracy of MRI with meningioma volume in (CC).

Methods: Prospective study of Saudi both males \& females patients of different age-randomly of 35 patients using CT. Scan axial, sagittal and coronal scans while in MRI Scan using standard protocol of examinations $\mathrm{T} 1$ weighted image and T2 weighted image in all cases were examined. The results are classified in tables and using ChiSquare analysis.

Results: Both CT. Scan and MRI exam's can find meningioma lesion more than zero (cc). The table -7 Chi-Square test showing the P. Value $=0.410>0.05$ showing No. significant between the CT. Scan and MRI in finding the meningioma.

Moreover, the table-10: Shows Chi-Square the P.value $=0.008>0.05$ showing significant in assessment of meningioma volume in (cc).

Conclusion: Meningioma CT. Scan is useful examination in emergency patients. While MRI should always be considered as an indispensable imaging modality in the assessment of meningioma volume (cc). Both CT. Scan and MRI are used to support the diagnosis and evaluation of meningioma. But MRI is better in accurate diagnosis meningioma volume (cc).

Copy Right, IJAR, 2018,. All rights reserved.

\section{Introduction:-}

The brain is an important organ that controls thought, memory, emotion, touch, motor skills, vision, respiration, body temperature, hunger, and many other processes that regulate our body. The brain has three main parts: Cerebrum, cerebellum and brainstem. There are two types of primary brain tumors: Benign tumor and malignant tumor. While meningioma is the kind of tumor( usually benign )starts in the outer linings of the brain (meninges). It is more common in adults. Many meningiomas can be removed with surgery, but some may grow back. ${ }^{(2,4,8)}$. With

Corresponding Author:-Hisham Al-nutayfi.

Address:-X-ray department, Al-Aflaj General Hospital M.O.H, Kingdom of Saudi Arabia. 
the increasing availability and use of modern brain diagnostic imaging modalities, has become fairly common. Although meningiomas are considered a type of primary brain tumor, they do not grow from brain tissue itself, but instead arise from the meninges, three thin layers of tissue covering the brain and spinal cord ${ }^{(5)}$. These tumors most commonly grow inward causing pressure on the brain or spinal cord, but they may also grow outward toward the skull, causing it to thicken. Most meningiomas are benign, slow-growing tumors. Some contain cysts (sacs of fluid), calcifications (mineral deposits), or tightly packed bunches of blood vessels ${ }^{(6)}$. There are several systems used to name, or group, these tumors. One system names meningioma by the type of cells in the tumor ${ }^{(9)}$. Syncytial (or meningothelial) meningiomas are the most common and feature unusually plump cells. Fibroblastic meningioma feature long, thin shaped cells ${ }^{(10)}$. Transitionalmeningiomas contain both types of cells. Another system uses the terms benign, atypical and malignant (or anaplastic) to describe the overall grade of meningioma ${ }^{(16)}$. In this system, benign meningioma contain easily recognized, well-differentiated (resembling normal) cell types which tend to grow slowly ${ }^{(11)}$. Atypical tumors represent $10-15 \%$ of meningioma. They contain proliferating cells that maybe faster growing and more likely to grow back after treatment, even after seemingly complete resection (surgical removal) ${ }^{(15)}$. Therefore, these tumors must be followed carefully for early signs of recurrence. Malignant or "anaplastic" tumors are poorly differentiated forms that often recur rapidly. Although they are quite rare (1-3\%), malignant meningioma scan be highly aggressive and difficult to treat. Another common practice is to attach the location of the tumor to its name. For example, a parasagittal meningioma is located near the sagittal sinus, a major blood vessel at the top of the cerebral hemispheres ${ }^{(12)}$. A sphenoid ridge meningioma is found along the ridge of bone behind the eyes and nose. Some meningioma scans cause problems despite their benign nature, because they are difficult to remove when they are located in functionally sensitive or hard to reach areas. Depending on the situation, stereotactic radiotherapy or radiosurgery may be particularly helpful in some of these cases. ${ }^{(18)}$ Although MRI is the imaging study of choice for evaluation of suspected meningioma or in the context of known or highly suspected pathology, computed tomography (CT) is more widely available, is better suited for rapid screening in urgent settings, and can be used when patients have MRI exclusions (such as pacemakers). As such, many meningiomas are first encountered on CT scans obtained for different reasons ${ }^{(13)}$. CT has a place in the diagnosis of meningioma because it is superior in demonstrating the effects of this neoplasm on adjacent bone, specifically osseous destruction in atypical or malignant meningiomas or hyperostosis associated with the benign meningiomas, and is more sensitive in detecting psammomatous calcifications in the tumor. ${ }^{(7)}$ The image contrast was assessed as good or excellent in plain CT and in plain MRI, but in the contrast-enhanced CT and MRI studies respectively. Adequate tumour delineation was achieved in plain CT, in plain MRI of the contrast-enhanced CT and MRI examinations. The contrast-enhanced studies proved to be superior to the plain CT and MRI studies as regards image contrast and tumor delineation. Because of the methodological advantages of the MRI technique, contrastenhanced MRI was judged to be slightly superior to contrast-enhanced CT. ${ }^{(2)}$ Magnetic resonance imaging (MRI) with gadolinium contrast enhancement in defining the histologic margins of brain tumors ${ }^{(14)}$. compared to conventional computed tomography (CT) using iodinated contrast enhancement. All tumors were visualized with contrast-enhanced MRI which proved most sensitive $\left.{ }^{(17}\right)$. Gadolinium bright enhancement of tumors in a distribution that consistently corresponded to areas of pathologically proved tumor infiltration. MRI is more accurate than unenhanced MRI, unenhanced CT, or enhanced CT in defining the histologic margins of tumors. ${ }^{(1)}$

\section{Materials and Methods:-}

Prospective experimental field research practical study of Saudi patients of different age were examined at King Khalid University Hospital Riyadh K.S.A-randomly of 35 patients at period of 9/2018- 11/2018, most patients complain of brain meningioma complaints of low back pain and leg weakness since brain tumors can mimic other diagnoses and present in conjunction with other musculoskeletal disorders. We selected patients whom have MRI request from their consultant physician with previous history of CT. Scan examination to confirm or for better evaluation or or have CT. Scan request we follow up whom confirmed by MRI exam. Patient preparation for brain CT scan, we should need to know the following: If the patient has allergies to iodine, medications, or food. Any previous reactions to contrast media. If the patient has asthma. The last LMP for female whether there is any possibility of pregnancy. If the patient has diabetes for scheduling him early in the morning. We explain the procedure to the patient. If the procedure involves the use of contrast dye, consent should be signed form the patient need fasting and. Patient to have the MRI exam and whether the exam needs to be modified for your particular condition. Pacemaker, Pregnancy, Claustrophobia, Implanted drug infusion device (i.e., insulin pump). Metallic implants and allergy to iodine, or gadolinium. Patient generally, there is no fasting requirement prior to a CT or MRI scan skull, unless a contrast dye is to be used. may be asked not to eat or drink anything for up to 4 hours before the scan and should arrive at least 30 minutes prior to your exam and check in with the receptionist. MRI contrast is indicated for the exam; an IV catheter will be inserted in your arm by a nurse. The duration of the procedure will 
vary but the average is 45 minutes to one hour per body part. The basic types of sequences used in brain MRI create either T1-weighted or T2-weighted images. While in C.T. Scan procedure was positioning patient in supine where head first arms be side with basic projections of axial and sagittal had been be taken with CT slice thickness 3-5 $\mathrm{mm}$. The results classified in tables and using Chi-Square analysis.

\section{Results:-}

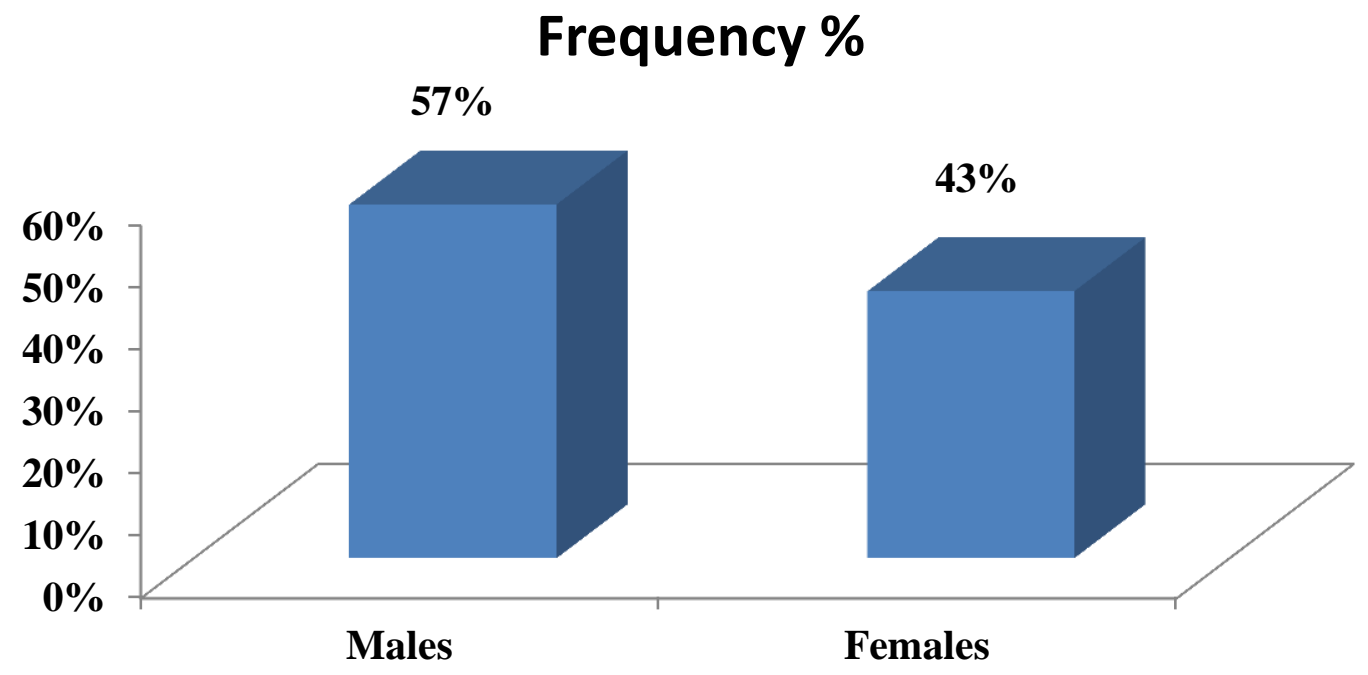

Fig 1:-Shows the sex distribution of patients $(\mathrm{n}=35)$

\section{Frequency \%}

$28.50 \%$

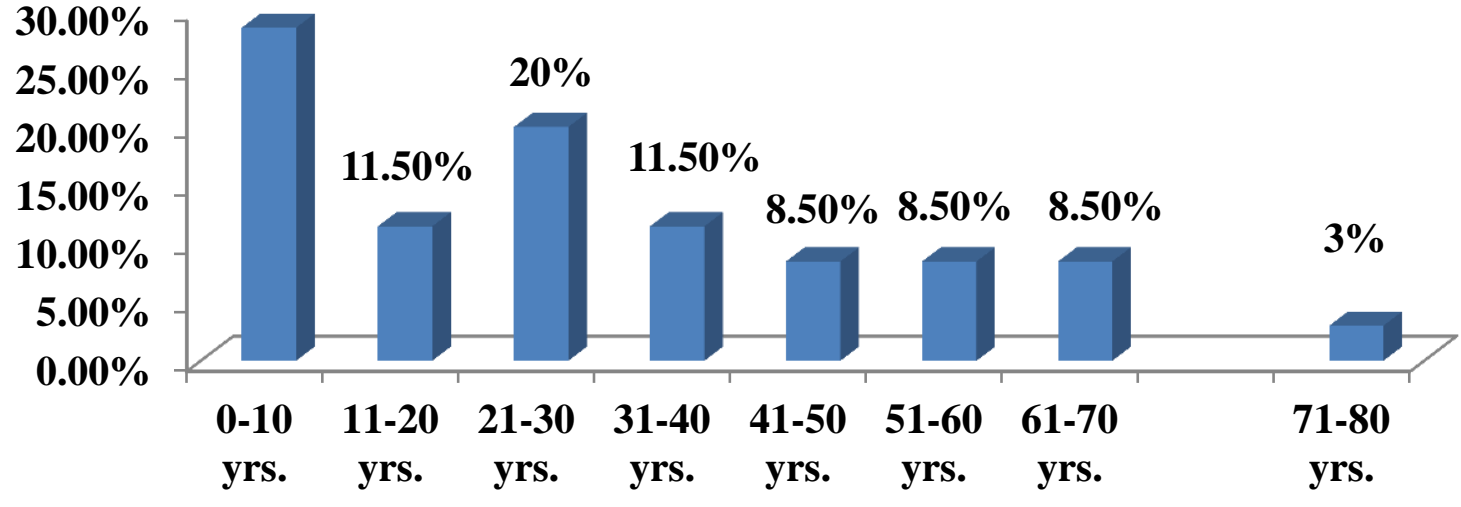

Fig 2:-Shows the distribution of patients according to age groups $(\mathrm{n}=35)$ 


\section{Frequency\%}

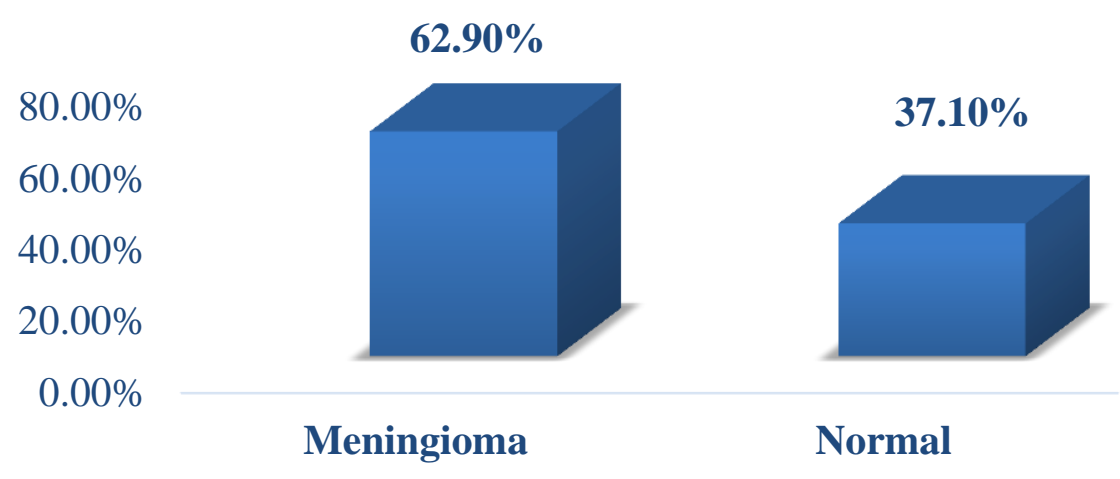

Fig 3:-show distribution of patients according to result.

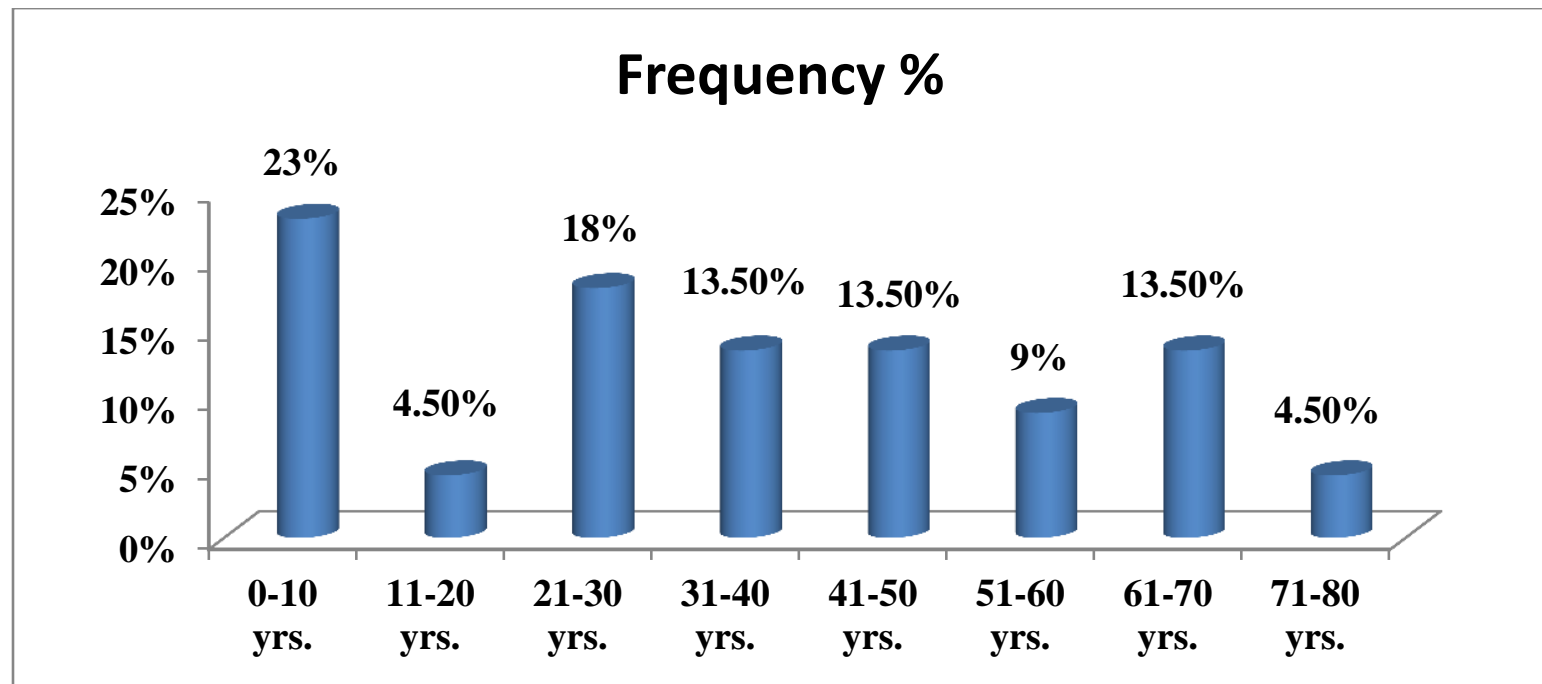

Fig 4:-Shows the distribution of patients according the positive findings $(n=22)$

\section{Compersion volume in CT ASCAN AND MRI}

20

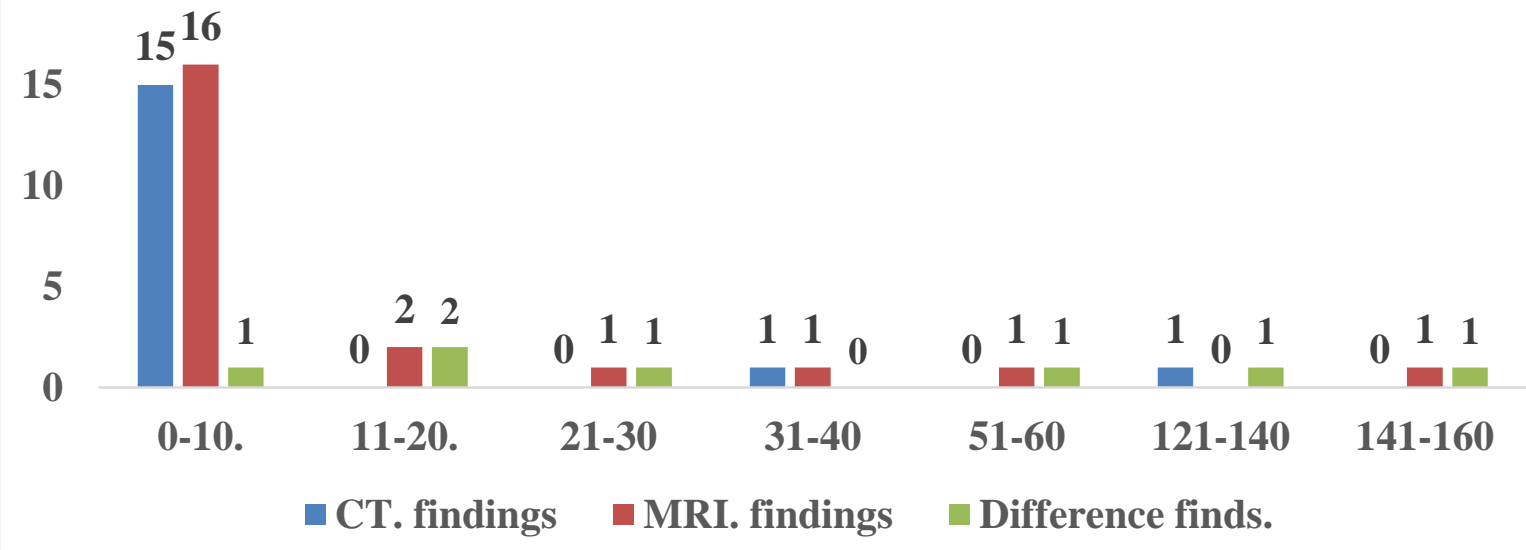

Fig 6:-Show volume in CT scan, MRI examination and difference 


\section{Positive in frequency \%}

\section{$100 \%$}

$77.20 \%$

$100.00 \%$
$80.00 \%$
$60.00 \%$
$40.00 \%$
$20.00 \%$
$0.00 \%$
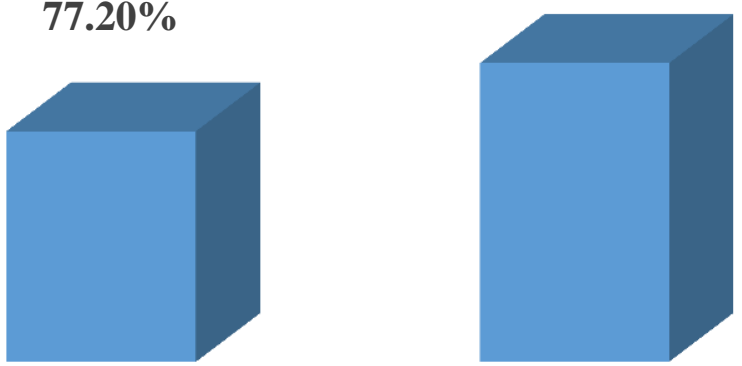

CT scan

MRI

Fig 6:-shows distribution of positive findings according to each modality of investigation.

Table 1:-Shows the paired Samples Chi-Square-Test for meningioma volume (cc)

\begin{tabular}{|c|c|c|c|c|c|c|c|c|c|}
\hline \multicolumn{10}{|c|}{ Paired Samples Test } \\
\hline & & \multicolumn{5}{|c|}{ Paired Differences } & \multirow[t]{3}{*}{$\mathrm{t}$} & \multirow[t]{3}{*}{ df } & \multirow{3}{*}{$\begin{array}{l}\text { Sig. } \\
(2- \\
\text { tailed })\end{array}$} \\
\hline & & \multirow[t]{2}{*}{ Mean } & \multirow[t]{2}{*}{$\begin{array}{l}\text { Std. } \\
\text { Deviation }\end{array}$} & \multirow[t]{2}{*}{$\begin{array}{l}\text { Std. } \\
\text { Error } \\
\text { Mean }\end{array}$} & \multicolumn{2}{|c|}{$\begin{array}{l}95 \% \text { Confidence } \\
\text { Interval of the } \\
\text { Difference }\end{array}$} & & & \\
\hline & & & & & Lower & Upper & & & \\
\hline Pair 1 & $\begin{array}{l}\text { CT. } \\
\text { findings } \\
\text {-MRI. } \\
\text { findings }\end{array}$ & - & 12.56099 & 3.04649 & -9.03356 & 3.88297 & - & 16 & .410 \\
\hline
\end{tabular}

P.value $=0.410>0.05$

Not significant

Table 2:-Shows the differences meningioma volume (cc)

\begin{tabular}{|c|c|c|c|}
\hline Patient. No. & CT. Volume(CC) & MRI Volume(CC) & Difference (CC) \\
\hline 1 & 137.20 & 148.20 & 11.00 \\
\hline 2 & 0.13 & 1.00 & 0.87 \\
\hline 3 & 1.87 & 3.04 & 0.17 \\
\hline 4 & 0.12 & 0.13 & 0.10 \\
\hline 5 & 0.90 & 1.00 & 7.00 \\
\hline 6 & 8.00 & 15.00 & 0.17 \\
\hline 7 & 0.00 & 1.17 & 0.13 \\
\hline 8 & 0.00 & 0.13 & 27.10 \\
\hline 9 & 4.32 & 31.42 & 0.09 \\
\hline 10 & 0.03 & 0.12 & 7.00 \\
\hline 11 & 1.00 & 8.00 & 2.38 \\
\hline 12 & 1.00 & 3.38 & 0.87 \\
\hline 13 & 0.13 & 1.00 & 0.12 \\
\hline 14 & 0.00 & 0.12 & 1.61 \\
\hline 15 & 0.12 & 1.73 & 21.48 \\
\hline 16 & 0.12 & 21.60 & 0.40 \\
\hline 17 & 1.33 & 1.73 & 1.00 \\
\hline 18 & 0.00 & 1.00 & 5.16 \\
\hline 19 & 5.60 & 10.76 & \\
\hline
\end{tabular}




\begin{tabular}{|c|c|c|c|}
\hline 20 & 0.12 & 1.00 & 0.88 \\
\hline 21 & 0.00 & 1.00 & 1.00 \\
\hline 22 & 33.00 & 58.50 & 25.50 \\
\hline
\end{tabular}

Table 3:-Comparison between CT. Scan\& MRI in meningioma exam's

\begin{tabular}{|l|l|l|}
\hline Type of comparison & CT. Scan exam's & MRI exam's \\
\hline Ionizing radiation exposure & More & None \\
\hline Duration l time & Various & Real \\
\hline Cost of service & Expensive & Most expensive \\
\hline Accuracy & Acceptable & Most acceptable \\
\hline Examination appointment time & Short period time & Long period time \\
\hline
\end{tabular}

\section{Discussion:-}

Meningioma is most common in female than in males and more common on age from 1 year up to 30 years old and usually they are convexity meningioma in the brain. MRI scanning is currently the best diagnostic modality. This study was performed in 35 patients. A total of 35 patient's basis ranging age 2-74years (mean age $=38$ years) in Saudi population $20(57 \%)$ male and 15(43\%) female (Figure.1). Distribution of patients according to age group (110years) 10 patients (28\%) are most examined while the group aage (71-80years) one patient (3\%) (Figure.2). Is the least examined were out of total 35 patients, $13(37.1 \%)$ patients are normal findings and other 22(62.9\%) patients are meningioma findings (Figure.3). Positive findings most effected the group age (1-10years) account 5 (23\%) and (21-30years) account $4(18 \%)$ while the least age group is $(71-80$ yeas $)=1(4.5 \%)$ show meningioma (Figure.4). In our examination 22patients whom positive. in the CT. Scan exam $17(77 \%)$ patients showing meningioma volume (cc) while 22patients whom positive, in MRI exam (100\%) showing meningioma volume(cc) (Figure.5\&6). ChiSquare test showing the P.value $=0.410>0.05$ showing No, significant between the CT. Scan and MRI in finding the meningioma (Table.1). The CT. Scan Showing the volume (cc) in $(17 / 22)=(77 \%)$ positive meningioma patients while the MRI showing the volume $(\mathrm{cc})$ in $(22 / 22)=(100)$ positive meningioma. Moreover, table-10: Shows ChiSquare the P.value $=0.008>0.05$ showing significant(Table.2). However, MRI is reliable for meningioma volume(cc) than CT. Scan according to our study this is matching the pervious study done by, Prabhakar et al 2007 they found, The mean and median volume of the tumor as measured from MR scans was $19.67 \mathrm{cc}+/-13.73$ and $16.13 \mathrm{cc}$ (range: $3.25 \mathrm{cc}$. The mean and median CM shift between CT and MR was $5.47 \mathrm{~mm}$ and $5.21 \mathrm{~mm}$ respectively ${ }^{(19)}$

\section{Conclusion:-}

Meningioma CT. Scan is useful examination. Fast aid emergency patients. While MRI should always be considered as an indispensable (trust) imaging modality in the assessment of meningioma volume (cc). Both CT. Scan and MRI are used to support the diagnosis and evaluation of meningioma. But MRI is better in accurate diagnosis meningioma volume than CT. Scan. Sultan Alshoabi et al $2015^{(20)}$ they found in their study, Meningioma is most common in female than in males and more common after 40 years old age and usually they are convexity meningioma in the brain. MRI scanning is currently the best diagnostic modality for detecting the brain and spine meningiomas it provides excellent detail regarding to the site, size, shape, intensity and enhancement after contrast administration.

\section{References:-}

1. Whelan HT, Clanton JA, Wilson RE\& Tulipan NB, (1988, Sep-Oct ). Comparison of CT and MRI brain tumor imaging using a canine glioma model. Pediatr Neurol, 4(5),279-83.

2. Schörner W, "et al.," (1990). Intracranial meningiomas. Comparison of plain and contrast-enhanced examinations in CT and MRI. Neuroradiology,32(1),12-8.

3. Chen C.,"et al.," (2007). Histopathology of Radiation Necrosis with Severe Peritumoral Edema after Gamma Knife Radiosurgery for Parasagittal Meningioma . Stereotact Funct Neurosurg ,85,292-295. https://doi.org/10.1159/000107362

4. Prabhakar R1, Haresh KP, Ganesh T, Joshi RC, Julka PK\&Rath GK,( 2007).

5. GBD 2015 Disease and Injury Incidence and Prevalence, Collaborators. (8 October 2016). "Global, regional, and national incidence, prevalence, and years lived with disability for 310 diseases and injuries, 1990-2015: a systematic analysis for the Global Burden of Disease Study 2015". Lancet.388 (10053): 15451602. doi:10.1016/S0140-6736(16)31678-6. PMC 5055577. PMID 27733282 
6. Amit Mittal, Kennith F. Layton, Sam Finn, George. Snipes, and Michael J. Opatowsky, Cystic meningioma: unusual imaging appearance of a common intracranial tumor, Proc (Bayl Univ Med Cent). 2010 Oct; 23(4): $429-431$

7. Saloner, A. Uzelac, S. Hetts, A. Martin, \& W. Dillon( 2010 ,Sep). Modern meningioma imaging techniques. J Neurooncol,99(3), 333-340.. doi: 10.1007/s11060-010-0367-6

8. Chamoun R, Krisht KM\&Couldwell WT, (2011) . Incidental meningiomas. Neurosurg Focus, 31(6),E19. doi: 10.3171/2011.9.FOCUS11220.

9. Marta GN, Correa SF\&Teixeira MJ (2011). Meningioma: review of the literature with emphasis on the approach to radiotherapy. Expert Rev Anticancer Ther. 11(11),1749-58. doi: 10.1586/era.11.162.

10. Winward Choy, B.A., "etal", (2011. The molecular genetics and tumor pathogenesis of meningiomas and the future directions of meningioma treatments. Neurosurg Focus,30 (5),

11. Koech, J. Orege, F. Ndiangui,B. Macharia, and N. Mbaruku(2013). Multiple Intracranial Meningiomas: A Review of the Literature and a Case Report. Hindawi Publishing Corporation Case Reports in Surgery, Article ID 131962, 4pages.http://dx.doi.org/10.1155/2013/131962.

12. Watts, G. Box, A. Galvin, P. Brotchie, N. Trost, and T. Sutherland (2014, Feb) Magnetic resonance imaging of meningiomas: A pictorial review. Insights Imaging,5(1), 113 -122. doi: 10.1007/s13244-013-0302-4.

13. Fu Y, Sun ZH, Zhang J\&Liu HT,(2015).Cystic meningioangiomatosis with enhancing mural nodule on MRI and no calcification on CT. Neurol Neurochir Pol,49(1),58-60. doi: 10.1016/j.pjnns.2014.12.001. Epub 2014 Dec 11.

14. Sun Z, "et al.,"( 2015). Three cases of sporadic meningioangiomatosis with different imaging appearances: case report and review of the literature. World J Surg Oncol. 28,13-89. doi: 10.1186/s12957-015-0477-x4.

15. Akira Kunimatsu , "et al.,"( 2016). Variants of meningiomas: a review of imaging findings and clinical features .Japanese Journal of Radiology, 34, ( 7) ,pp 459-469.

16. Benita Tamrazi, Mark S. Shiroishi and Chia-Shang J. Liu ( 2016) . Advanced Imaging of Intracranial Meningiomas. Neurosurg Clin N Am, 27(2), 137-143. doi:10.1016/j.nec.2015.11.004.

17. Mehdorn HM (2016). Intracranial Meningiomas: A 30-Year Experience and Literature Review. Adv Tech Stand Neurosurg. (43),139-84. doi: 10.1007/978-3-319-21359-0_6.

18. https:// www.abta.org . Copy right $@ 2017$.

19. The mean and median volume of the tumor as measured from MR scans was $19.67 \mathrm{cc}+/-13.73$ and $16.13 \mathrm{cc}$ (range: $3.25 \mathrm{cc}$. The mean and median CM shift between CT and MR was $5.47 \mathrm{~mm}$ and $5.21 \mathrm{~mm}$ respectively

20. Sultan Alshoabi, "Moawia Gameraddin, Suliman Salih1, Jumaa Tamboul1, Fathelrehman Alagab,Suzan Abdelmaboud, Evaluation of Meningioma of the brain and spine with Computerized Tomography and Magnetic Resonance Imaging, April 2015 with 117 Reads Taibah University King Saudi Arabia. 